\title{
THE LEGENDRE-TAU TECHNIQUE FOR THE DETERMINATION OF A SOURCE PARAMETER IN A SEMILINEAR PARABOLIC EQUATION
}

ABBAS SAADATMANDI, MEHDI DEHGHAN, AND ANTONIO CAMPO

Received 20 April 2005; Revised 10 November 2005; Accepted 20 November 2005

A numerical procedure for an inverse problem concerning diffusion equation with source control parameter is considered. The proposed method is based on shifted Legendre-tau technique. Our approach consists of reducing the problem to a set of algebraic equations by expanding the approximate solution as a shifted Legendre function with unknown coefficients. The operational matrices of integral and derivative together with the tau method are then utilized to evaluate the unknown coefficients of shifted Legendre functions. Illustrative examples are included to demonstrate the validity and applicability of the presented technique.

Copyright (C) 2006 Abbas Saadatmandi et al. This is an open access article distributed under the Creative Commons Attribution License, which permits unrestricted use, distribution, and reproduction in any medium, provided the original work is properly cited.

\section{Introduction}

In this paper, we will consider an inverse problem of finding a source parameter $p(t)$ in the following semilinear parabolic equation:

$$
u_{t}=u_{x x}+p(t) u+q(x, t), \quad 0 \leq x \leq \ell, 0<t \leq \tau,
$$

with initial condition

$$
u(x, 0)=f(x), \quad 0 \leq x \leq \ell
$$

and boundary conditions

$$
\begin{array}{ll}
u(0, t)=g_{0}(t), & 0<t \leq \tau, \\
u(\ell, t)=g_{1}(t), & 0<t \leq \tau,
\end{array}
$$

Hindawi Publishing Corporation

Mathematical Problems in Engineering

Volume 2006, Article ID 70151, Pages 1-11

DOI 10.1155/MPE/2006/70151 
and subject to the overspecification over a portion of the spatial domain

$$
\int_{0}^{s(t)} u(x, t) d x=k(t), \quad 0<t \leq \tau, 0<s(t)<\ell,
$$

where $f, g_{0}, g_{1}, s, q$, and $k$ are known functions, while the functions $u(x, t)$ and $p(t)$ are unknown.

The existence and uniqueness and continuous dependence of the solution to this problem is discussed in $[1-5,15,18]$.

Certain types of physical problems can be modeled by (1.1)-(1.4). For example, if $u$ represents a temperature distribution, then (1.1)-(1.4) can be interpreted as the control problem with source control. We want to identify the function $p(t)$ that will yield a desired energy prescribed in a portion of the spatial domain. This kind of problem has many important applications [13, 17, 20, 22].

The presence of an integral term in a boundary condition can greatly complicate the application of standard numerical techniques such as finite difference, finite elements, spectral methods, and so forth. It is therefore important to be able to convert the nonclassical boundary value problems to a more desirable form, to make them more widely applicable to problems of practical interest. In many cases, this is a hard task $[7,12]$.

Several numerical procedures for solution of the problem (1.1)-(1.4) are given in [3, 9-11]. Recent publications also involved finite-difference approach [8]. In this work a different approach is used. Our approach consists of reducing the problem to a set of algebraic equations by expanding the approximate solution $u$ and $p$ as a shifted Legendre function with unknown coefficients. The operational matrices of integral and derivative are given. These matrices together with the tau method are then utilized to evaluate the unknown coefficients of shifted Legendre functions. The tau method was invented by Lanczos [16]. Recently there has been work on applications of the tau method published in literature, for instance, see $[16,19,21]$. The method consists of expanding the required approximate solution as the elements of a complete set of orthogonal functions. In the tau method, unlike the Galerkin approximation, the expansion functions are not required to satisfy the boundary constraint individually [6].

The rest of this paper is structured in the following way. In Section 2, we describe the basic formulation of shifted Legendre polynomials required for our subsequent development. Section 3 summarizes the application of shifted Legendre-tau method to the solution of problem (1.1)-(1.4). As a result a set of algebraic equations is formed and a solution of the considered problem is introduced. In Section 4, the proposed method is applied to three numerical examples. Section 5 ends this paper with a brief summary.

\section{Properties of shifted Legendre polynomials}

The well-known Legendre polynomials are defined on the interval $[-1,1]$ and can be determined with the aid of the following recurrence formulae:

$$
\begin{gathered}
L_{0}(z)=1, \quad L_{1}(z)=z, \\
L_{i+1}(z)=\frac{2 i+1}{i+1} z L_{i}(z)-\frac{i}{i+1} L_{i-1}(z), \quad i=1,2, \ldots
\end{gathered}
$$


In order to use these polynomials on the interval $[0, h]$, we define the so-called shifted Legendre polynomials by introducing the change of variable

$$
z=\frac{2 x-h}{h}, \quad 0 \leq x \leq h
$$

The shifted Legendre polynomials in $x$ are then obtained as follows:

$$
\begin{gathered}
L_{0}^{h}(x)=1, \quad L_{1}^{h}(x)=\frac{2 x-h}{h}, \\
L_{i+1}^{h}(x)=\frac{(2 i+1)(2 x-h)}{(i+1) h} L_{i}^{h}(x)-\frac{i}{i+1} L_{i-1}^{h}(x), \quad i=1,2, \ldots .
\end{gathered}
$$

The orthogonality condition is

$$
\int_{0}^{h} L_{i}^{h}(x) L_{j}^{h}(x) d x= \begin{cases}\frac{h}{2 i+1} & \text { for } i=j \\ 0 & \text { for } i \neq j\end{cases}
$$

A function $u(x, t)$ of two independent variables defined for $0 \leq x \leq \ell$ and $0 \leq t \leq \tau$ may be expanded in terms of double shifted Legendre polynomials as

$$
u(x, t)=\sum_{i=0}^{n} \sum_{j=0}^{m} a_{i j} L_{i}^{\tau}(t) L_{j}^{\ell}(x)=\Psi^{T}(t) \mathbf{A} \Phi(x),
$$

where the shifted Legendre coefficient matrix $\mathbf{A}$ and the shifted Legendre vectors $\Phi(x)$ and $\Psi(t)$ are given by

$$
\begin{gathered}
\mathbf{A}=\left(\begin{array}{ccc}
a_{00} & \cdots & a_{0 m} \\
\vdots & & \vdots \\
a_{n 0} & \cdots & a_{n m}
\end{array}\right), \\
\Phi(x)=\left[L_{0}^{\ell}(x), L_{1}^{\ell}(x), \ldots, L_{m}^{\ell}(x)\right]^{T}, \\
\Psi(t)=\left[L_{0}^{\tau}(t), L_{1}^{\tau}(t), \ldots, L_{n}^{\tau}(t)\right]^{T} .
\end{gathered}
$$

The derivative of the vector $\Phi(x)$ can be expressed by

$$
\frac{d \Phi(x)}{d x}=\mathbf{D} \Phi(x)
$$

where $\mathbf{D}$ is the $(m+1) \times(m+1)$ operational matrix of derivative given by

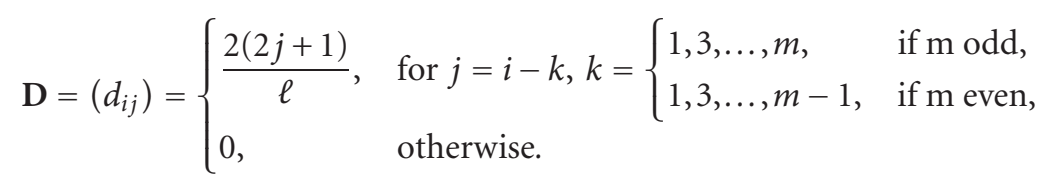


4 Legendre-tau technique for inverse parabolic equations

For example, for odd $m$, we have

$$
\mathbf{D}=\frac{2}{\ell}\left(\begin{array}{cccccccc}
0 & 0 & 0 & 0 & \cdots & 0 & 0 & 0 \\
1 & 0 & 0 & 0 & \cdots & 0 & 0 & 0 \\
0 & 3 & 0 & 0 & \cdots & 0 & 0 & 0 \\
1 & 0 & 5 & 0 & \cdots & 0 & 0 & 0 \\
\vdots & \vdots & \vdots & \vdots & \vdots & \vdots & \vdots & \vdots \\
0 & 3 & 0 & 7 & \cdots & 2 m-3 & 0 & 0 \\
1 & 0 & 5 & 0 & \cdots & 0 & 2 m-1 & 0
\end{array}\right)
$$

The shifted Legendre polynomials satisfy the following recurrence relationship [6]:

$$
\dot{L}_{r+1}^{h}(t)-\dot{L}_{r-1}^{h}(t)=\frac{2(2 r+1)}{h} L_{r}^{h}(t), \quad r=1,2, \ldots
$$

in which - denotes the derivative with respect to $t$.

Integrating (2.12) from 0 to $t$, we have

$$
\int_{0}^{t} L_{r}^{h}\left(t^{\prime}\right) d t^{\prime}=\frac{h}{2 r+1}\left[L_{r+1}^{h}(t)-L_{r-1}^{h}(t)\right], \quad r=1,2, \ldots
$$

In view of (2.13), the integration of $\Psi(t)$ defined in (2.8) from 0 to $t$ can be approximated by

$$
\int_{0}^{t} \Psi\left(t^{\prime}\right) d t^{\prime} \simeq \mathbf{P} \Psi(t),
$$

where $\mathbf{P}$ is an $(n+1) \times(n+1)$ operational matrix of integration given as

$$
\mathbf{P}=\tau\left(\begin{array}{ccccc}
\delta_{0} & \delta_{0} & & & \\
-\delta_{1} & 0 & \delta_{1} & & \\
& \ddots & \ddots & \ddots & \\
& & -\delta_{N-1} & 0 & \delta_{N-1} \\
& & & -\delta_{N} & 0
\end{array}\right)
$$

with $\delta_{k}=1 / 2(2 k+1)$.

Obviously similar to (2.14) we have

$$
\int_{0}^{x} \Phi\left(x^{\prime}\right) d x^{\prime} \simeq \mathrm{G} \Phi(x)
$$

where $\mathbf{G}$ is an $(m+1) \times(m+1)$ matrix and is defined similar to $(2.15)$. 


\section{The shifted Legendre-tau method}

Integrating (1.1) from 0 to $t$ and using (1.2) we have

$$
u(x, t)-f(x)=\int_{0}^{t} u_{x x}\left(x, t^{\prime}\right) d t^{\prime}+\int_{0}^{t} p\left(t^{\prime}\right) u\left(x, t^{\prime}\right) d t^{\prime}+\int_{0}^{t} q\left(x, t^{\prime}\right) d t^{\prime} .
$$

Similar to (2.5) we expand $q(x, t)$ as

$$
q(x, t)=\Psi^{T}(t) \mathbf{Q} \Phi(x)
$$

where $\mathbf{Q}$ is an $(n+1) \times(m+1)$ known matrix.

Expanding $f(x)$ by $(m+1)$ terms of shifted Legendre series, we get

$$
f(x)=\sum_{k=0}^{m} f_{k} L_{k}^{\ell}(x)=\Psi^{T}(t) \mathbf{F} \Phi(x)
$$

where $\mathbf{F}$ is a known $(n+1) \times(m+1)$ matrix and can be shown by

$$
\mathbf{F}=\left(\begin{array}{ccccc}
f_{0} & f_{1} & \cdots & f_{m-1} & f_{m} \\
0 & 0 & \cdots & 0 & 0 \\
\vdots & \vdots & \cdots & \vdots & \vdots \\
0 & 0 & \cdots & 0 & 0
\end{array}\right)
$$

The function $p(t)$ may be expanded in terms of $n+1$ shifted Legendre series as

$$
p(t)=\sum_{k=0}^{n} b_{k} L_{k}^{\tau}(t)=B^{T} \Psi(t)
$$

where $B^{T}=\left[b_{0}, \ldots, b_{n}\right]^{T}$ is an unknown vector.

Using (2.5), (2.9), and (2.14), we get

$$
\int_{0}^{t} u_{x x}\left(x, t^{\prime}\right) d t^{\prime}=\left(\int_{0}^{t} \Psi^{T}\left(t^{\prime}\right) d t^{\prime}\right) \mathbf{A}\left(\frac{d^{2} \Phi(x)}{d x^{2}}\right)=\Psi^{T}(t) \mathbf{P}^{T} \mathbf{A D}^{2} \Phi(x),
$$

and by using (2.5), (2.14), and (3.2) we have

$$
\int_{0}^{t} q\left(x, t^{\prime}\right) d t^{\prime}=\left(\int_{0}^{t} \Psi^{T}\left(t^{\prime}\right) d t^{\prime}\right) \mathbf{A} \Phi(x)=\Psi^{T}(t) \mathbf{P}^{T} \mathbf{A} \Phi(x) .
$$

Employing (2.5), (2.14), and (3.5), we get

$$
\int_{0}^{t} p\left(t^{\prime}\right) u\left(x, t^{\prime}\right) d t^{\prime}=\left(\int_{0}^{t} B^{T} \Psi\left(t^{\prime}\right) \Psi^{T}\left(t^{\prime}\right) d t^{\prime}\right) \mathbf{A} \Phi(x) .
$$

Now suppose

$$
w_{k, j, i}=\int_{0}^{\tau} L_{k}^{\tau}(t) L_{j}^{\tau}(t) L_{i}^{\tau}(t) d t, \quad k, j, i=0,1, \ldots, n
$$


6 Legendre-tau technique for inverse parabolic equations

by using (3.9) and (2.4) we can easily see that

$$
B^{T} \Psi(t) \Psi^{T}(t)=\Psi^{T}(t) \mathbf{H}
$$

where $\mathbf{H}$ is an $(n+1) \times(n+1)$ matrix given as

$$
\mathbf{H}_{i j}=\left(\frac{2 i+1}{\tau}\right) \cdot \sum_{k=0}^{n} b_{k} w_{k, j, i}, \quad i, j=0,1, \ldots, n .
$$

By using (3.10) and (2.14), (3.8) can be written as

$$
\int_{0}^{t} p\left(t^{\prime}\right) u\left(x, t^{\prime}\right) d t^{\prime}=\Psi^{T}(t) \mathbf{P}^{T} \mathbf{H A} \Phi(x) .
$$

Applying (2.5), (3.3), (3.6), (3.7), and (3.12), the residual $R(x, t)$ for (3.1) can be written as

$$
R(x, t)=\Psi^{T}(t)\left[\mathbf{A}-\mathbf{F}-\mathbf{P}^{T} \mathbf{A D}^{2}-\mathbf{P}^{T} \mathbf{H} \mathbf{A}-\mathbf{P}^{T} \mathbf{Q}\right] \Phi(x)=\Psi^{T}(t) \mathbf{E} \Phi(x),
$$

where

$$
\mathbf{E}=\mathbf{A}-\mathbf{F}-\mathbf{P}^{T} \mathbf{A D}^{2}-\mathbf{P}^{T} \mathbf{H A}-\mathbf{P}^{T} \mathbf{Q} .
$$

As in a typical tau method we generate $(n+1) \times(m-1)$ algebraic equations by using the following algebraic equations:

$$
\mathbf{E}_{i j}=0, \quad i=0, \ldots, n, j=0, \ldots, m-2 .
$$

Also, by substituting (2.5) in (1.3), we get

$$
\begin{aligned}
& \Psi^{T}(t) \mathbf{A} \Phi(0)=g_{0}(t), \\
& \Psi^{T}(t) \mathbf{A} \Phi(1)=g_{1}(t),
\end{aligned}
$$

respectively. Furthermore, applying (2.5) and (2.16) in (1.4), we get

$$
\Psi^{T}(t) \operatorname{AG} \Phi(s(t))=k(t) .
$$

Equations (3.16) and (3.17) are collocated at $n+1$ points. For suitable collocation points, we use the shifted Legendre roots $t_{i}, i=1, \ldots, n+1$, of $L_{n+1}^{\tau}(t)$. The number of the unknown coefficients $a_{i j}$ and $b_{i}$ is equal to $(n+1)(m+1)+(n+1)$ and can be obtained from (3.15), (3.16), and (3.17). Consequently $u(x, t)$ given in (2.5) and $p(t)$ given in (3.5) can be calculated.

\section{Numerical tests}

To give a clear overview of our technique, we have chosen three test problems. These examples are chosen such that there exist exact solutions for them. So the numerical methods described in previous sections were applied to the following examples. 
Table 4.1. Computational results for $u(x, 0.5)$ from Test 1 .

\begin{tabular}{cccccc}
\hline$x$ & $\begin{array}{c}\text { FTCS } \\
{[8]}\end{array}$ & $\begin{array}{c}\text { BTCS } \\
{[8]}\end{array}$ & $\begin{array}{c}\text { Crank- } \\
\text { Nicolson }[8]\end{array}$ & $\begin{array}{c}\text { Peresent method } \\
m=n=5\end{array}$ & $m=n=7$ \\
\hline 0.1 & $3.1 \times 10^{-3}$ & $4.8 \times 10^{-3}$ & $4.2 \times 10^{-3}$ & $2.8 \times 10^{-4}$ & $1.0 \times 10^{-6}$ \\
0.2 & $3.1 \times 10^{-3}$ & $4.6 \times 10^{-3}$ & $4.1 \times 10^{-3}$ & $1.5 \times 10^{-4}$ & $6.2 \times 10^{-6}$ \\
0.3 & $2.8 \times 10^{-3}$ & $4.4 \times 10^{-3}$ & $3.9 \times 10^{-3}$ & $2.0 \times 10^{-4}$ & $3.9 \times 10^{-6}$ \\
0.4 & $2.8 \times 10^{-3}$ & $4.3 \times 10^{-3}$ & $3.7 \times 10^{-3}$ & $2.3 \times 10^{-4}$ & $1.0 \times 10^{-6}$ \\
0.5 & $2.6 \times 10^{-3}$ & $4.1 \times 10^{-3}$ & $3.5 \times 10^{-3}$ & $1.4 \times 10^{-4}$ & $4.2 \times 10^{-6}$ \\
0.6 & $2.4 \times 10^{-3}$ & $4.1 \times 10^{-3}$ & $3.2 \times 10^{-3}$ & $4.9 \times 10^{-4}$ & $7.1 \times 10^{-6}$ \\
0.7 & $2.6 \times 10^{-3}$ & $4.2 \times 10^{-3}$ & $3.2 \times 10^{-3}$ & $4.1 \times 10^{-4}$ & $3.3 \times 10^{-6}$ \\
0.8 & $2.7 \times 10^{-3}$ & $4.3 \times 10^{-3}$ & $3.5 \times 10^{-3}$ & $3.4 \times 10^{-4}$ & $9.3 \times 10^{-6}$ \\
0.9 & $2.9 \times 10^{-3}$ & $4.5 \times 10^{-3}$ & $3.6 \times 10^{-3}$ & $2.4 \times 10^{-4}$ & $1.2 \times 10^{-6}$ \\
\hline
\end{tabular}

Test 1. Consider (1.1)-(1.4) with $\ell=1, \tau=0.5$, and

$$
\begin{gathered}
q(x, t)=\left(\pi^{2}+2 t\right) \exp (t) \cos (\pi x)+2 \exp (t) x t, \\
g_{0}(t)=\exp (t), \\
g_{1}(t)=0, \\
f(x)=x+\cos (\pi x), \\
s(t)=0.5(1+\sqrt{t}), \\
k(t)=\exp (t)\left(\frac{\sin (0.5 \pi(1+\sqrt{t}))}{\pi}+\frac{(1+\sqrt{t})^{2}}{8}\right),
\end{gathered}
$$

for which the exact solution is [8]

$$
\begin{gathered}
u^{*}(x, t)=\exp (t)(\cos (\pi x)+x), \\
p^{*}(t)=1-2 t
\end{gathered}
$$

In [8] the domain $[0, \ell] \times[0, \tau]$ is divided into an $M \times N$ mesh with the spatial step size $h=\ell / M$ in $x$ direction and the time step size $k=\tau / N$, respectively. Then the author used some finite-difference formulas such as classical FTCS formulas and BTCS scheme and Crank-Nicolson technique to solve (1.1)-(1.4). For the purpose of comparison in Tables 4.1 and 4.2, we compare the absolute error of our method with $m=n=5$ and $m=n=7$ together with the methods given in [8]. From Tables 4.1 and 4.2 we see that the Legendretau method is clearly reliable if compared with the finite-difference technique.

Also the absolute errors obtained for $u(x, 0.5)$ and $p(t)$ computed for $n=4$ and $m=$ 4, 6, 8 using the tau method discussed in Section 3 together with exact solution are shown in Tables 4.3 and 4.4, receptively. From Tables 4.3 and 4.4 we see that the approximation solution computed by fixed $n$ and different values of $m$ converges to the exact solution. 
8 Legendre-tau technique for inverse parabolic equations

Table 4.2. Computational results for $p(t)$ from Test 1 .

\begin{tabular}{lccccc}
\hline$t$ & FTCS & BTCS & Crank- & Peresent method & $m=n=7$ \\
& {$[8]$} & {$[8]$} & Nicolson $[8]$ & $m=n=5$ & \\
\hline 0.1 & $5.3 \times 10^{-3}$ & $6.9 \times 10^{-3}$ & $6.3 \times 10^{-3}$ & $9.0 \times 10^{-3}$ & $4.4 \times 10^{-5}$ \\
0.2 & $5.2 \times 10^{-3}$ & $6.8 \times 10^{-3}$ & $6.1 \times 10^{-3}$ & $2.3 \times 10^{-3}$ & $3.1 \times 10^{-5}$ \\
0.3 & $5.0 \times 10^{-3}$ & $6.6 \times 10^{-3}$ & $5.8 \times 10^{-3}$ & $4.0 \times 10^{-3}$ & $7.4 \times 10^{-5}$ \\
0.4 & $4.9 \times 10^{-3}$ & $6.4 \times 10^{-3}$ & $5.6 \times 10^{-3}$ & $2.8 \times 10^{-3}$ & $2.8 \times 10^{-5}$ \\
0.5 & $4.7 \times 10^{-3}$ & $6.3 \times 10^{-3}$ & $5.3 \times 10^{-3}$ & $1.5 \times 10^{-2}$ & $3.2 \times 10^{-4}$ \\
\hline
\end{tabular}

Table 4.3. Absolute errors for $u(x, 0.5)$ with $n=4$ and $m=4,6,8$ from Test 1.

\begin{tabular}{ccccc}
\hline$x$ & Exact & $\begin{array}{c}m=4 \\
\text { Error }\end{array}$ & $\begin{array}{c}m=6 \\
\text { Error }\end{array}$ & $\begin{array}{c}m=8 \\
\text { Error }\end{array}$ \\
\hline 0.1 & 1.732899 & $7.8 \times 10^{-3}$ & $2.0 \times 10^{-4}$ & $2.0 \times 10^{-6}$ \\
0.2 & 1.663588 & $2.3 \times 10^{-2}$ & $3.6 \times 10^{-5}$ & $1.2 \times 10^{-6}$ \\
0.3 & 1.463710 & $2.8 \times 10^{-2}$ & $3.5 \times 10^{-4}$ & $1.4 \times 10^{-6}$ \\
0.4 & 1.168971 & $1.8 \times 10^{-2}$ & $4.0 \times 10^{-4}$ & $4.1 \times 10^{-6}$ \\
0.5 & 0.824361 & $5.2 \times 10^{-4}$ & $3.6 \times 10^{-5}$ & $6.1 \times 10^{-6}$ \\
0.6 & 0.479750 & $1.9 \times 10^{-2}$ & $3.3 \times 10^{-4}$ & $2.9 \times 10^{-6}$ \\
0.7 & 0.185011 & $2.9 \times 10^{-2}$ & $2.9 \times 10^{-4}$ & $2.3 \times 10^{-6}$ \\
0.8 & -0.014867 & $2.4 \times 10^{-2}$ & $7.8 \times 10^{-5}$ & $2.4 \times 10^{-6}$ \\
0.9 & -0.084178 & $9.2 \times 10^{-3}$ & $2.2 \times 10^{-4}$ & $7.7 \times 10^{-6}$ \\
\hline
\end{tabular}

Now we define the maximum errors for $u(x, t)$ and $p(t)$ as

$$
\begin{gathered}
e_{u}=\left\|u_{n, m}-u^{*}\right\|_{\infty}=\max \left\{\left|u_{n, m}(x, t)-u^{*}(x, t)\right|, 0<x \leq \ell, 0<t \leq \tau\right\}, \\
e_{p}=\left\|p_{n, m}-p^{*}\right\|_{\infty}=\max \left\{\left|p_{n, m}(t)-p^{*}(t)\right|, 0<x \leq \ell, 0<t \leq \tau\right\},
\end{gathered}
$$

respectively, where $u_{n, m}(x, t)$ and $p_{n, m}(t)$ are the computed result with $n$ and $m$. In Table 4.5 we give the maximum errors $e_{u}$ and $e_{p}$ for $n=4,5,6,7$ and $m=n$.

From Table 4.5 we see that the errors decrease rapidly as $n$ and $m$ increase.

Test 2. Consider (1.1)-(1.4) with $\ell=1, \tau=0.5$, and

$$
\begin{aligned}
q(x, t)= & \left(1-t^{3}\right) \sin (x)-x^{2}(t-1)^{2} \exp \left(t^{2}\right)-2 \exp \left(t^{2}\right)-t^{2}\left(\pi \cos (x)+t^{3}+t-3\right), \\
g_{0}(t)= & \pi+t^{3} \\
g_{1}(t)= & t \sin (1)+\exp \left(t^{2}\right)+\pi \cos (1)+t^{3}, \\
f(x)= & x^{2}+\pi \cos (x), \\
s(t)= & t+\sin (t), \\
k(t)= & (\pi \sin (t)-t \cos (t)) \cos (\sin (t))+(t \sin (t)+\pi \cos (t)) \sin (\sin (t)) \\
& +\frac{1}{3} \exp \left(t^{2}\right)\left(\sin ^{3}(t)+t^{3}\right)+\left(t^{2} \sin (t)+t \sin ^{2}(t)\right) \exp \left(t^{2}\right)+\left(1+t^{3}+t^{2} \sin (t)\right) t,
\end{aligned}
$$


Abbas Saadatmandi et al. 9

Table 4.4. Absolute errors for $p(t)$ with $n=4$ and $m=4,6,8$ from Test 1 .

\begin{tabular}{lcccc}
\hline$t$ & Exact & $\begin{array}{c}m=4 \\
\text { Error }\end{array}$ & $\begin{array}{c}m=6 \\
\text { Error }\end{array}$ & $\begin{array}{c}m=8 \\
\text { Error }\end{array}$ \\
\hline 0.05 & 0.900000 & $2.1 \times 10^{-1}$ & $1.8 \times 10^{-4}$ & $3.1 \times 10^{-6}$ \\
0.10 & 0.800000 & $4.6 \times 10^{-2}$ & $6.6 \times 10^{-4}$ & $8.3 \times 10^{-6}$ \\
0.15 & 0.700000 & $2.3 \times 10^{-2}$ & $7.4 \times 10^{-4}$ & $6.4 \times 10^{-6}$ \\
0.20 & 0.600000 & $5.2 \times 10^{-2}$ & $5.4 \times 10^{-5}$ & $2.5 \times 10^{-6}$ \\
0.25 & 0.500000 & $7.1 \times 10^{-2}$ & $3.8 \times 10^{-4}$ & $5.2 \times 10^{-7}$ \\
0.30 & 0.400000 & $5.5 \times 10^{-2}$ & $4.0 \times 10^{-4}$ & $1.2 \times 10^{-5}$ \\
0.35 & 0.300000 & $8.2 \times 10^{-3}$ & $5.5 \times 10^{-4}$ & $2.5 \times 10^{-7}$ \\
0.40 & 0.200000 & $2.8 \times 10^{-2}$ & $6.2 \times 10^{-4}$ & $1.0 \times 10^{-6}$ \\
0.45 & 0.100000 & $1.8 \times 10^{-2}$ & $2.3 \times 10^{-4}$ & $1.9 \times 10^{-6}$ \\
0.50 & 0.000000 & $2.5 \times 10^{-1}$ & $1.2 \times 10^{-3}$ & $1.4 \times 10^{-5}$ \\
\hline
\end{tabular}

Table 4.5. The maximum errors for $e_{u}$ and $e_{p}$ for different values of $n$ from Test 1 .

\begin{tabular}{ccccc}
\hline$n$ & 4 & 5 & 6 & 7 \\
\hline$e_{u}$ & $3.3 \times 10^{-2}$ & $6.0 \times 10^{-4}$ & $4.0 \times 10^{-4}$ & $7.0 \times 10^{-6}$ \\
$e_{p}$ & $6.5 \times 10^{-1}$ & $2.7 \times 10^{-2}$ & $3.4 \times 10^{-3}$ & $4.4 \times 10^{-4}$ \\
\hline
\end{tabular}

Table 4.6. The maximum errors for $e_{u}$ and $e_{p}$ for different values of $n$ from Test 2 .

\begin{tabular}{cccccc}
\hline$n$ & 3 & 4 & 5 & 6 & 7 \\
\hline$e_{u}$ & $7.0 \times 10^{-3}$ & $2.1 \times 10^{-4}$ & $1.9 \times 10^{-5}$ & $2.9 \times 10^{-7}$ & $2.4 \times 10^{-8}$ \\
$e_{p}$ & $5.6 \times 10^{-2}$ & $2.1 \times 10^{-3}$ & $1.1 \times 10^{-4}$ & $4.1 \times 10^{-6}$ & $8.1 \times 10^{-8}$ \\
\hline
\end{tabular}

for which the exact solution is

$$
\begin{gathered}
u^{*}(x, t)=t \sin (x)+x^{2} \exp \left(t^{2}\right)+\pi \cos (x)+t^{3}, \\
p^{*}(t)=t^{2}+1 .
\end{gathered}
$$

The maximum errors $e_{u}$ and $e_{p}$ for $n=3,4,5,6,7$ and $m=n$ are shown in Table 4.6.

Test 3. Consider (1.1)-(1.4) with $\ell=1, \tau=0.5$, and

$$
\begin{aligned}
q(x, t)= & x \exp (t)\left(1-t^{2}-\sin (t)\right)+\exp (t)\left(\sin (t)+\cos (t)-t^{2} \sin (t)-\sin ^{2}(t)\right) \\
& +x^{2} t\left(2-t^{3}-t \sin (t)\right)-2 t^{2} \\
g_{0}(t)= & \exp (t) \sin (t) \\
g_{1}(t)= & (1+\sin (t)) \exp (t)+t^{2} \\
f(x)= & x \\
s(t)= & t+\sqrt{t} \\
k(t)= & \frac{1}{6}(t+\sqrt{t})\left(2 t^{3}(t+1)+4 t^{7 / 2}+3 \exp (t)(t+\sqrt{t}+2 \sin (t))\right)
\end{aligned}
$$


10 Legendre-tau technique for inverse parabolic equations

Table 4.7. The maximum errors for $e_{u}$ and $e_{p}$ for different values of $n$ from Test 3 .

\begin{tabular}{cccccc}
\hline$n$ & 3 & 4 & 5 & 6 & 7 \\
\hline$e_{u}$ & $6.1 \times 10^{-5}$ & $6.2 \times 10^{-6}$ & $1.7 \times 10^{-7}$ & $3.4 \times 10^{-9}$ & $6.2 \times 10^{-11}$ \\
$e_{p}$ & $4.5 \times 10^{-4}$ & $2.8 \times 10^{-5}$ & $5.6 \times 10^{-7}$ & $1.3 \times 10^{-8}$ & $1.6 \times 10^{-9}$ \\
\hline
\end{tabular}

which is easily seen to have the exact solution

$$
\begin{gathered}
u^{*}(x, t)=(x+\sin (t)) \exp (t)+x^{2} t^{2}, \\
p^{*}(t)=t^{2}+\sin (t) .
\end{gathered}
$$

In Table 4.7 we give the maximum errors $e_{u}$ and $e_{p}$ for $n=3,4,5,6,7$ and $m=n$.

From Tables 4.6 and 4.7 we see that the errors decrease rapidly as $n$ and $m$ increase. It is clear that in Tests 1, 2, and 3 the Legendre-tau method can be considered as an efficient method.

\section{Conclusion}

In this paper, we presented a numerical scheme for solving the one-dimensional inverse parabolic problem. Our approach was based on the shifted Legendre-tau method. The stability and convergence of the Legendre-tau approximations (see [14]) make this approach very attractive and contributed to the good agreement between approximate and exact values for the numerical tests. The obtained results showed that this approach can solve the problem effectively. The new described computational technique produces very accuracy results even when employing a small number of collocation points.

\section{Acknowledgment}

The authors are very grateful to the anonymous referee for carefully reading the paper and for his/her comments and suggestions which have improved the paper.

\section{References}

[1] B. M. Budak and A. D. Iskenderov, A class of inverse boundary value problems with unknown coefficients, Soviet Mathematics Doklady 8 (1967), 1026-1029.

[2] J. R. Cannon and Y. P. Lin, An inverse problem of finding a parameter in a semi-linear heat equation, Journal of Mathematical Analysis and Applications 145 (1990), no. 2, 470-484.

[3] J. R. Cannon, Y. Lin, and S. Wang, Determination of source parrameter in parabolic equations, Meccanica 27 (1992), no. 2, 85-94.

[4] J. R. Cannon, Y. P. Lin, and S. Xu, Numerical procedures for the determination of an unknown coefficient in semi-linear parabolic differential equations, Inverse Problems 10 (1994), no. 2, 227243.

[5] J. R. Cannon and H.-M. Yin, Numerical solutions of some parabolic inverse problems, Numerical Methods for Partial Differential Equations 6 (1990), no. 2, 177-191.

[6] C. Canuto, M. Y. Hussaini, A. Quarteroni, and T. A. Zang, Spectral Methods in Fluid Dynamics, Springer Series in Computational Physics, Springer, New York, 1988. 
[7] M. Dehghan, Finite difference schemes for two-dimensional parabolic inverse problem with temperature overspecification, International Journal of Computer Mathematics 75 (2000), no. 3, 339349.

[8] __ An inverse problem of finding a source parameter in a semilinear parabolic equation, Applied Mathematical Modelling 25 (2001), no. 9, 743-754.

[9] - Fourth-order techniques for identifying a control parameter in the parabolic equations, International Journal of Engineering Science 40 (2002), 433-447.

[10] - Determination of a control function in three-dimensional parabolic equations, Mathematics and Computers in Simulation. 61 (2003), no. 2, 89-100.

[11] _ Numerical computation of a control function in a partial differential equation, Applied Mathematics and Computation 147 (2004), no. 2, 397-408.

[12] Efficient techniques for the second-order parabolic equation subject to nonlocal specifications, Applied Numerical Mathematics 52 (2005), no. 1, 39-62.

[13] _ Parameter determination in a partial differential equation from the overspecified data, Mathematical and Computer Modelling 41 (2005), no. 2-3, 196-213.

[14] D. Gottlieb, M. Y. Hussaini, and S. A. Orszag, Theory and applications of spectral methods, Spectral Methods for Partial Differential Equations (Hampton, Va, 1982) (R. Voigt, D. Gottlieb, and M. Hussaini, eds.), SIAM, Pennsylvania, 1984, pp. 1-54.

[15] B. F. Jones Jr., The determination of a coefficient in a parabolic differential equation. I. Existence and uniqueness, Journal of Mathematics and Mechanics 11 (1962), 907-918.

[16] C. Lanczos, Applied Analysis, Prentice-Hall, New Jersey, 1956.

[17] J. A. MacBain, Inversion theory for a parameterized diffusion problem, SIAM Journal on Applied Mathematics 47 (1987), no. 6, 1386-1391.

[18] A. I. Prilepko and V. V. Solov'ev, On the solvability of inverse boundary value problems for the determination of the coefficient preceding the lower derivative in a parabolic equation, Differential Equations 23 (1987), no. 1, 136-143 (Russian).

[19] M. Razzaghi, S. Oppenheimer, and F. Ahmad, Tau method approximation for radiative transfer problems in a slab medium, Journal of Quantitative Spectroscopy and Radiative Transfer $\mathbf{7 2}$ (2002), no. 4, 439-447.

[20] W. Rundell, Determination of an unknown nonhomogeneous term in a linear partial differential equation from overspecified boundary data, Applicable Analysis 10 (1980), no. 3, 231-242.

[21] A. Saadatmandi and M. Razzaghi, A tau method approach for the diffusion equation with nonlocal boundary conditions, International Journal of Computer Mathematics 81 (2004), no. 11, 14271432.

[22] S. Wang and Y. P. Lin, A finite-difference solution to an inverse problem for determining a control function in a parabolic partial differential equation, Inverse Problems 5 (1989), no. 4, 631-640.

Abbas Saadatmandi: Department of Mathematics, Kashan University, Kashan, Iran

E-mail address: saadatmandi@kashanu.ac.ir

Mehdi Dehghan: Department of Applied Mathematics, Faculty of Mathematics and Computer Science, Amirkabir University of Technology, 424 Hafez Avenue, Tehran 15914, Iran E-mail address: mdehghan@aut.ac.ir

Antonio Campo: Department of Mechanical Engineering, The University of Vermont, Burlington, VT 05405, USA

E-mail address: acampo@cem.uvm.edu 


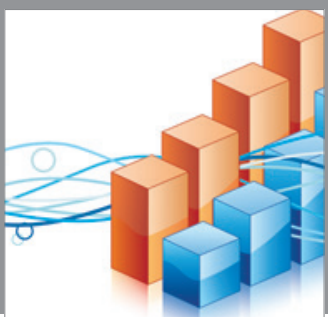

Advances in

Operations Research

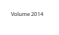

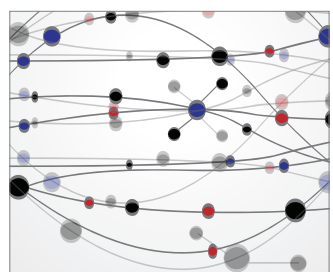

\section{The Scientific} World Journal
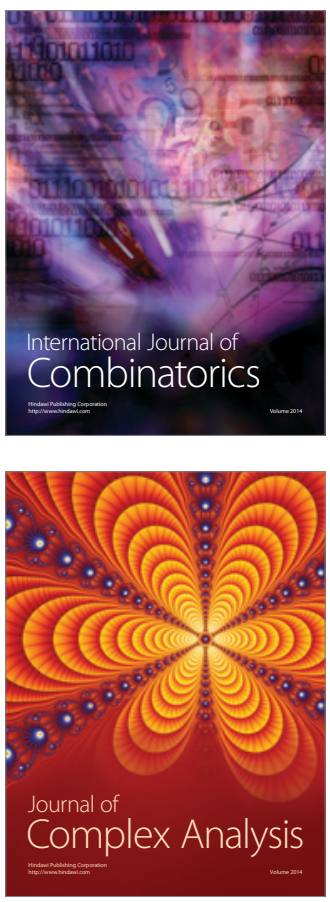

International Journal of

Mathematics and

Mathematical

Sciences
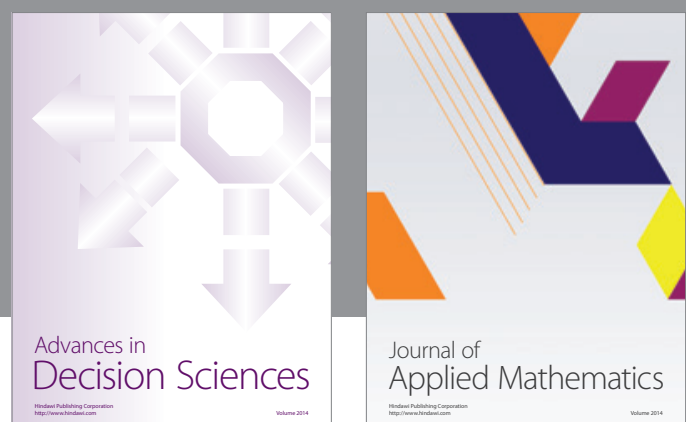

Journal of

Applied Mathematics
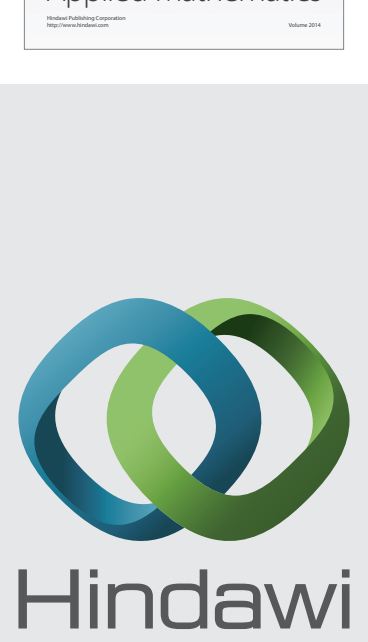

Submit your manuscripts at http://www.hindawi.com
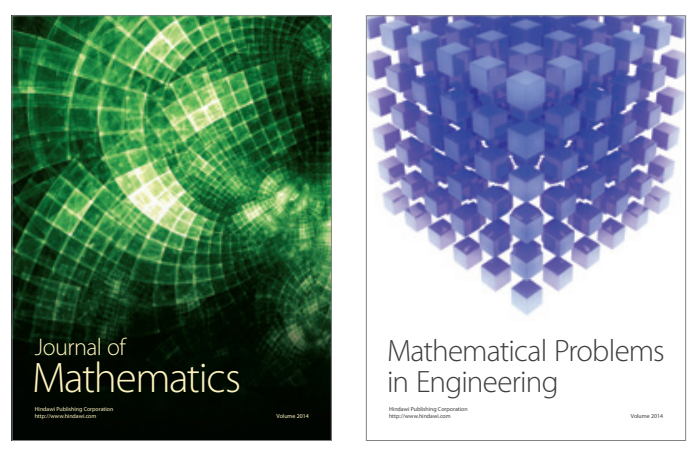

Mathematical Problems in Engineering
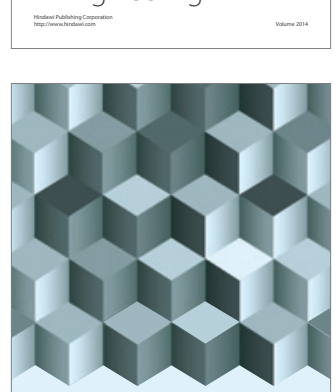

Journal of

Function Spaces
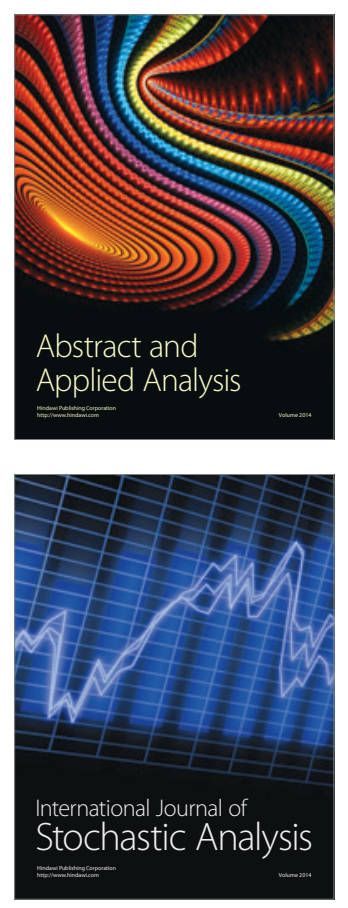

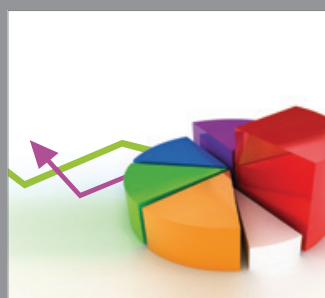

ournal of

Probability and Statistics

Promensencen
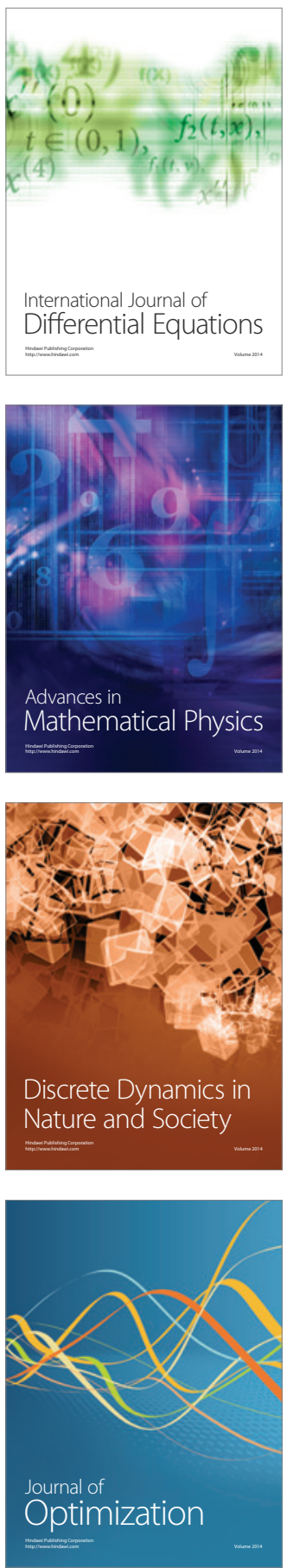